\title{
Thermal diffusivity measurement of mural painting by simulated infra-red thermography
}

\author{
J.L Bodnar*, J.L. Nicolas*, K. Mouhoubi*, V. Detalle** \\ *GRESPI / ECATHERM, UFR Sciences Exactes et Naturelles, BP 1039, 51687 Reims cedex 02, France \\ ** Laboratoire de Recherche des Monuments Historiques, 29 avenue du Paris, 77420 Champs sur Marne, France
}

\begin{abstract}
In this work, we present a method of in situ measurement, of longitudinal thermal diffusivity of work of art. We present first the principle of this measurement method. It is based on the temporal analysis of the characteristic radius of the photothermal signature of a laser excitation. We present then the experimental device developed for the study. We show finally, experimentally, that the method allows a good estimation of the longitudinal thermal diffusivity of a sample test and of a partial copy of the "Saint Christophe" of the "Campana" collection of the "Louvre".
\end{abstract}

\section{Introduction}

Within the framework of the restoration of work of art, we work, since about ten years, to the detection of detachment and air pockets located in murals paintings. Stimulated infra-red thermography already allowed us to detect detachment located in the "Saint Christophe" of the "Campana" collection of the "Louvre", in the painted walls of the church "Saint Florentin" of "Bonnet", in the ceilings painted of the abbey of "Savin Saint sur Gartempe" (classified with the world inheritance of UNESCO) and finally in the "Cocteau" frescos of the church "Saint Pierre" of "Villefranche sur mer" [1-7]. These qualitative studies being positive, we now study the possibilities of the photothermal method for the defects characterization. To this end, the first thermophysical parameter we chose to study is the thermal diffusivity. Indeed, we hope on the one hand that this parameter will enable us to estimate the depth of the studied defect. We hope in addition that this parameter will enable us to characterize the pictorial layer and consequently will give information about the realization of the work of art. We hope finally, that the method will enable us to detect capillary salt ascent, but also to characterize them. To measure these thermal diffusivity parameter, we had two solutions. We could on the one hand, take a sample of work of art and implement a traditional measurement method of this thermophysical parameter (flash method [8-9]). We could in addition, to develop a method usable in situ and being non destructive for the studied mural painting. For reasons of preservation of the works of art, it is this last option which we chose to implement. The thickness of a mural painting being often equal to several tens of centimetres, a transverse thermal diffusivity measurement is often impossible. We thus chose to develop a longitudinal thermal diffusivity measurement of the work of art. It is this method which we present here. We present first the principle of this measurement method. It is based on the temporal analysis of the characteristic radius of the photothermal signature of a laser excitation. We present then the experimental device developed for the study. We show finally, experimentally, that the method allows a good estimation of the longitudinal thermal diffusivity of a sample test and of a partial copy of the "Saint Christophe" of the "Campana" collection of the "Louvre".

\section{The longitudinal thermal diffusivity measurement method developed for the study}

The principle of the longitudinal thermal diffusivity measurement method developed for the study is the following: A sample is lighted with a localised laser excitation. It is temporally close to a Dirac function $\delta(t)$. It is spatially close to a Gaussian shape. We measure then the variations of temperature induced by this excitation. We use an infra-red camera of thermography. From the 
temporal evolution of this field of temperature, we estimate, using a mathematical post processing, the wanted longitudinal thermal diffusivity. This mathematical post processing is based to the one initially presented by $\mathrm{F}$ Cernuschi et al. [10]. Let us examine in details this mathematical post processing. Let us consider a plate. Its thickness is equal to L. It is semi infinite radially. Let us consider a very short thermal excitation. It is close to a Dirac function $\delta(t)$. Its spatially shape is a Gaussian one. This excitation is applied at $\mathrm{t}=0$, in the centre of the preceding plate. $\mathrm{R}$ is the characteristic radius of the excitation spot (measured for $\mathrm{Q}_{\max } / \mathrm{e}^{2}$ ). $\lambda, \rho, \mathrm{c}$ and $\mathrm{a}$, are respectively, the thermal conductivity, the density, the heat capacity and the thermal diffusivity of the studied plate. The sample is initially in thermal balance with its environment. Finally, the model neglects the convecto - radiative exchanges between the studied sample and the environment. The mathematical transcription of these assumptions leads to the following differential system:

$$
\begin{gathered}
\Delta T(r, z, t)=\frac{1}{a} \cdot \frac{\partial T(r, z, t)}{\partial t} \\
\mathrm{Z}=0:-\lambda \frac{\partial T(z, 0, t)}{\partial t}=\frac{2 Q}{\pi R^{2}} \operatorname{Exp}\left(-\frac{2 r^{2}}{R^{2}}\right) \delta(t) \\
\mathrm{Z}=\mathrm{L}:-\lambda \frac{\partial T(z, L, t)}{\partial t}=0 \\
\mathrm{t}=0: \mathrm{T}=\mathrm{T}_{\text {env }}
\end{gathered}
$$

To solve this differential system, we chose to implement two integral transformations: The first one is a space transformation. It is an order 0 Hankel transformation with $\mathrm{r}$ axis. The second one is a temporal transformation. It is a Fourier transformation. By taking into account these integral transforms, the equation with the partial derivates becomes:

$$
\frac{\partial^{2} T(\sigma, z, \omega)}{\partial z^{2}}-\left(\sigma^{2}+\frac{i . \omega}{a}\right) T(\sigma, z, \omega)=0
$$

The solution of this partial derivate equation is then written in the transformed space:

$$
T(\sigma, z, \omega)=\frac{Q}{2 \pi \lambda} \frac{\exp \left(\frac{-\sigma^{2} R^{2}}{8}\right)}{\sqrt{\sigma^{2}+\frac{i . \omega}{a}}} \frac{\operatorname{ch}\left[(z-L) \sqrt{\left.\sigma^{2}+\frac{i . \omega}{a}\right]}\right.}{\operatorname{sh}\left(L \sqrt{\sigma^{2}+\frac{i . \omega}{a}}\right)}
$$

After inversing the integral transformations, we obtain the expression of the surface temperature of the analyzed sample:

$$
T(r, 0, t)=\frac{2 Q}{b \sqrt{\pi^{3} t}} \cdot \frac{1}{R^{2}+8 a t} \cdot \exp \left(-\frac{2 r^{2}}{R^{2}+8 a t}\right) \sum_{n=-\infty}^{n=+\infty} \exp \left\{-\frac{[(n-1) L]^{2}}{a t}\right\}
$$

Let us consider now our experimental conditions. It is the study of a plaster plate. Its thickness is equal to $1 \mathrm{~cm}$. Its thermal diffusivity is equal to $510^{-7} \mathrm{~m}^{2} / \mathrm{s}$. Let us consider in addition limits of calculation equal to -10000 and +10000 . With these conditions, the series intervening in the 
expression (4) is close to 1 during at least $50 \mathrm{~s}$ (for a more important thickness, the value of the series would be close to 1 during a longer time). This duration is quite higher than that of a complete analysis. Consequently, it is possible to neglect this series in the expression (4). We obtain then:

$$
T(r, 0, t) \approx \frac{2 Q}{b \sqrt{\pi^{3} t}} \cdot \frac{1}{R^{2}+8 a t} \cdot \exp \left(-\frac{2 r^{2}}{R^{2}+8 a t}\right)
$$

The wanted thermal diffusivity is then simply given starting from the slope of the temporal evolution of the characteristic radius (measured with $\mathrm{T}_{\max } / \mathrm{e}^{2}$ ) of the thermal signature of the laser excitation (6).

$$
r_{c}^{2}=R^{2}+8 a t
$$

\section{The experimental device implemented for the study}

The experimental device implemented for the study is the SAMMTHIR System of the Reims University. It is composed of three parts. It is composed on the one hand of an excitation source. It is a laser diode. Its emitting wavelength is equal $810 \mathrm{~nm}$. It is associated with an optic of collimation and with an optic of focusing. The experimental device is composed in addition of an optic of detection. It is an infrared camera of thermography. It is a "long waves" camera. It uses bolometer detectors. It is implemented in macro mode, to obtain a sufficient space resolution. It is placed perpendicularly with the studied sample. The pencil of light emitting by the laser diode, because of obstruction of the camera, lights the analysed sample with a tilted way. Its shape is then slightly elliptic. The experimental device is finally composed of electronic and data processing software. This last part allows piloting the laser diode. It allows then a controlled temporal shape of the excitation beam. It is a crenel one in our study. This last part allows finally the acquisition and the post treatment of the photothermal signals (figure 1).

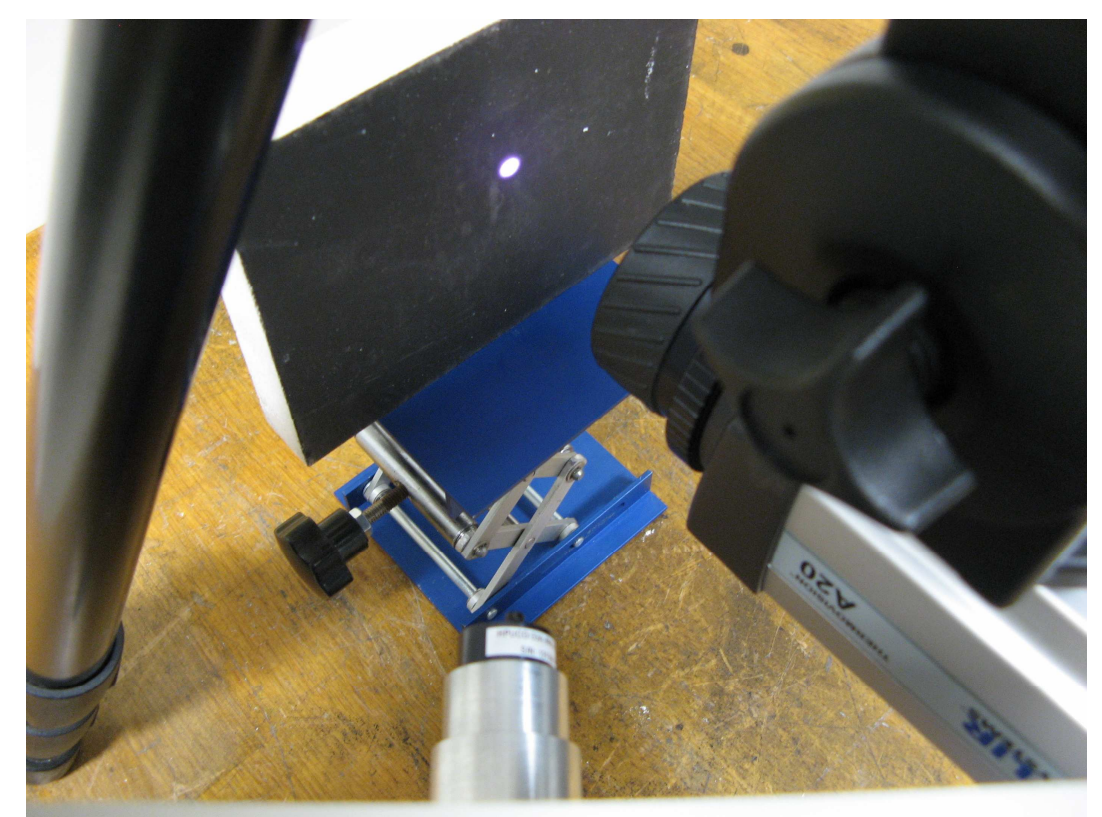

Figure 1: The experimental device implemented for the study 


\section{The experimental results obtained}

\subsection{Characterization of an academic sample}

In order to test experimentally the measurement protocol retained and the experimental device implemented, we initially tried to characterize an academic sample. It is a block of plaster. Its length is equal to $15 \mathrm{~cm}$. Its width is equal to $12 \mathrm{~cm}$. Its thickness is equal to $2.2 \mathrm{~cm}$. In order to simulate the presence of a pictorial layer; we covered it with a fine coat of black paint. We measured first his transverse thermal diffusivity using a flash diffusivimeter [8-9]. We found a value equal to $3.4910^{-7} \mathrm{~m}^{2} / \mathrm{s}$. We then studied it using SAMMTHIR system. The experimental conditions retained for the study are the followings: The distance between the infra-red camera of thermography and the studied sample is equal to $5 \mathrm{~cm}$. The power of excitation is equal to $2 \mathrm{~W}$. The duration of excitation is equal to $20 \mathrm{~ms}$. The frequency of analysis is equal to $50 \mathrm{~Hz}$. In the figure 2 , we present four thermograms representative of the temporal evolution of the photothermal signature of the laser spot. It shows as waited theoretically, that the photothermal signature becomes more diffuse and less intense with time.
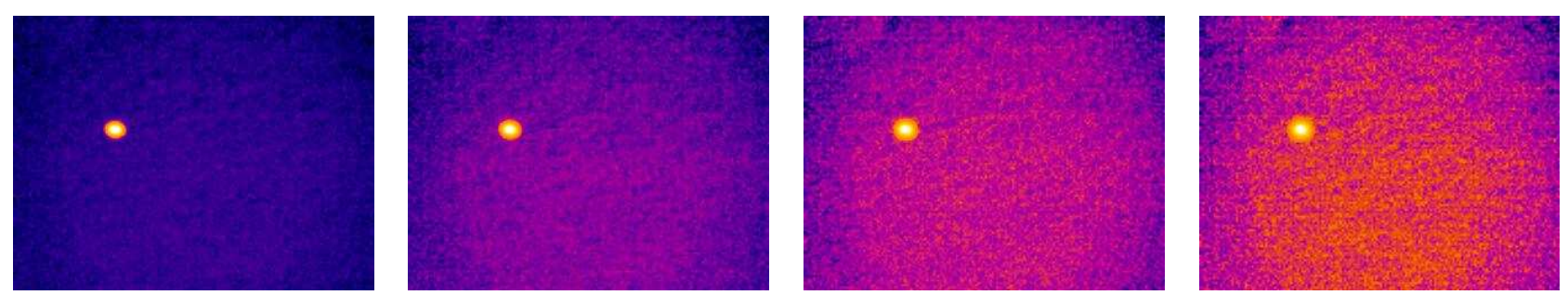

Figure 2: Temporal evolution of the photothermal signature of the laser heating

From these thermograms, we then drew, for each instant, the profiles of the photothermal signature of the excitation. From these profiles, we then measured the characteristic radius necessary to our study. In the figure 3, we drew the temporal evolution of this characteristic radius. We find as waited theoretically a line with a positive slope.

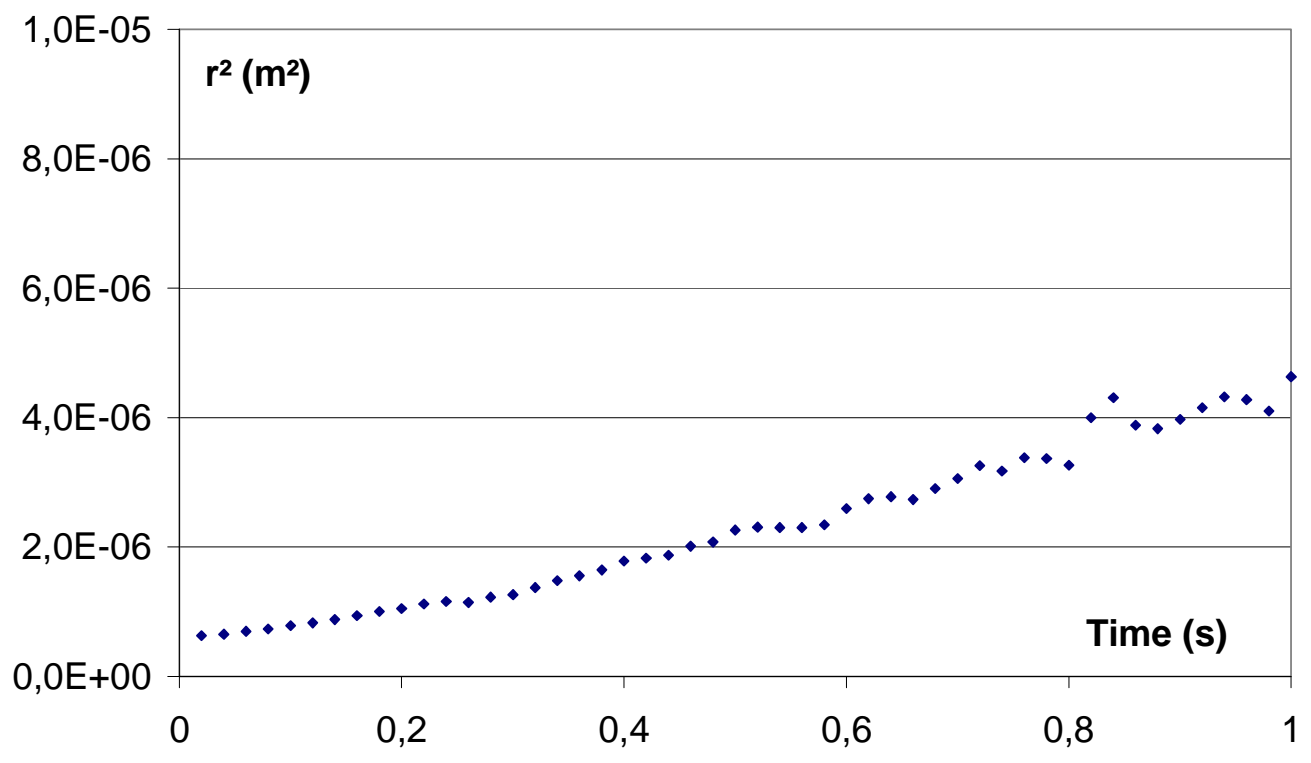

Figure 3: Temporal evolution of the characteristic radius of the photothermal signature of the laser spot 
We then calculated the value of this slope by adjusting a line to the experimental data. We estimated a slope equal to $2.8610^{-6} \mathrm{~m}^{2} / \mathrm{s}$. We finally used the formula (6), to calculate the wanted thermal diffusivity. We find then a value equal to $3.5710^{-7} \mathrm{~m}^{2} / \mathrm{s}$. The value measured with the flash diffusivimeter was equal to $3.49 .10^{-7} \mathrm{~m}^{2} / \mathrm{s}$. These two values are close. That confirms the possibilities of the photothermal method for longitudinal thermal diffusivity measurement.

\subsection{Characterization of an academic fresco}

The experimental results obtained previously being positive, we tried to characterize now an academic fresco. It is a partial copy of "Saint Christophe" of the "Campana" collection of the "Louvre". This mural was manufactured by Gabriela Szatanik. She is a professional restorer. She used the technique of the Italians primitive. It is manufactured in a mixture of lime and plaster deposited on a plaster substrate. The whole was covered "al fresco" with a pictorial layer representing the Jesus child (figure 4).

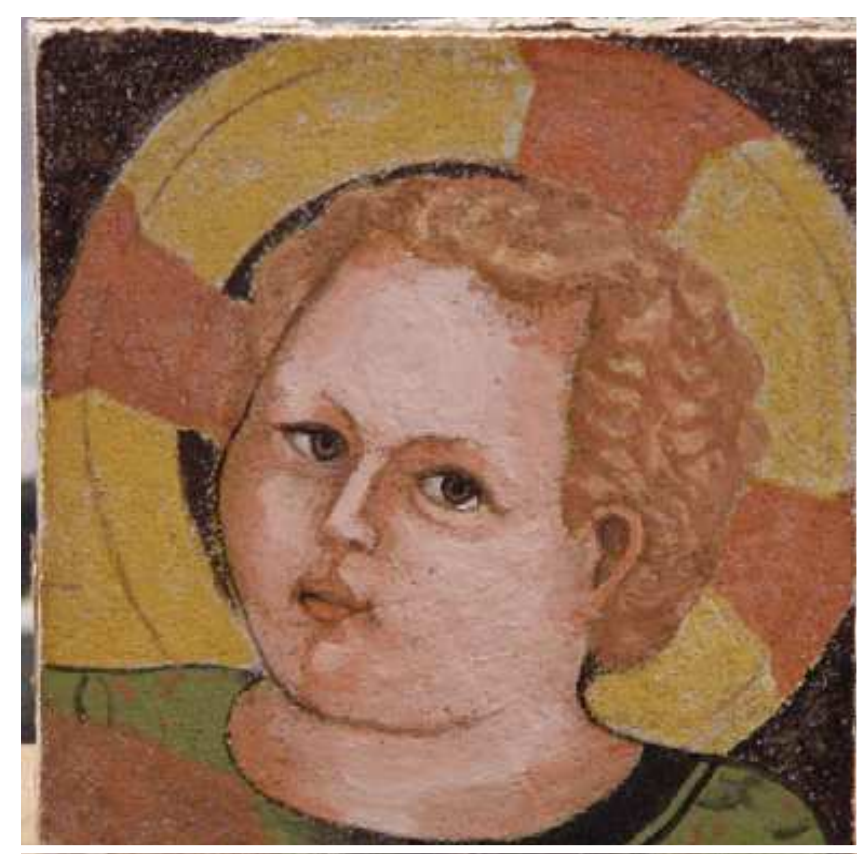

Figure 4: The academic fresco studied

This fresco already was the subject of many studies [1-7]. In particular, its longitudinal thermal diffusivity was already measured using a space Fourier analysis. Its estimated value was equal to $5.13 .10^{-7} \mathrm{~m}^{2} / \mathrm{s}$ [6-7]. In this Work, we studied it by taking into account experimental conditions close to those retained for the study of the preceding plaster sample. We considered the following adjustments: The distance between the sample and the infrared camera is equal to $7.5 \mathrm{~cm}$. The distance between the laser and the sample is equal to $10 \mathrm{~cm}$. The laser power is equal to $2 \mathrm{~W}$. The duration of excitation is equal to $1 \mathrm{~s}$. Finally the frequency of analysis is equal to $50 \mathrm{~Hz}$. The analyzed area is the left eye of the Jesus child (figure 5). 


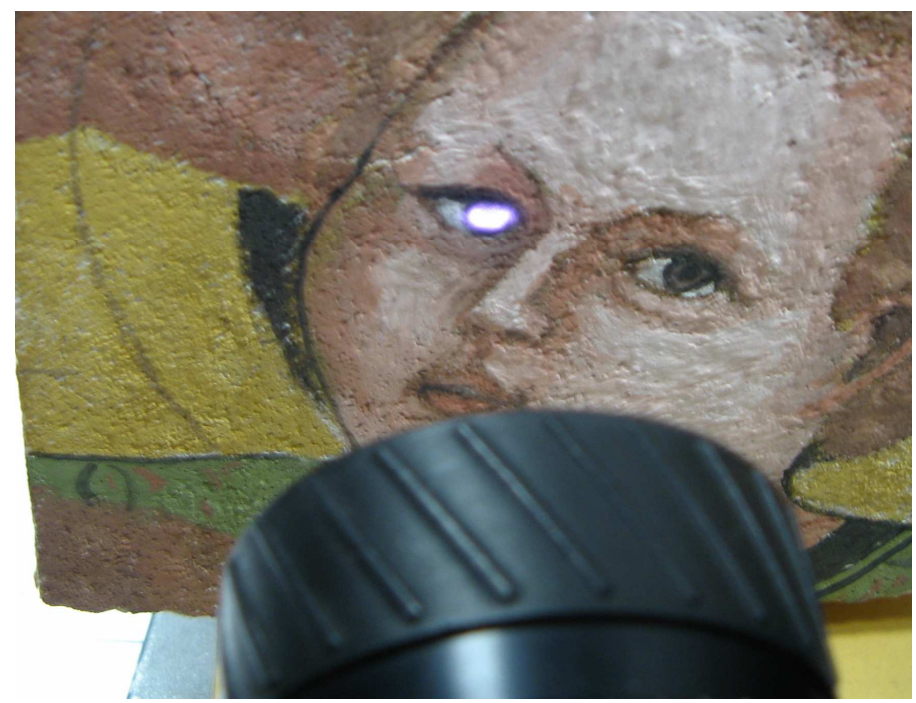

Figure 5: the analyzed area

As previously, we drew from the thermograms obtained for each instant, the profiles of the photothermal signature of the excitation. From these profiles, we then determined the characteristic radius necessary to our study. In the figure 6 , we drew the temporal evolution of this characteristic radius. We find like previously and like waited theoretically a line with a positive slope. We then estimated this slope. We estimated a slope equal to $4,07810^{-6} \mathrm{~m}^{2} \mathrm{~s}^{-1}$. We have finally, using the formula (6), to calculate the wanted longitudinal thermal diffusivity. We find then an average value equal to $5.0910^{-7} \mathrm{~m}^{2} / \mathrm{s}$. This value is close to that obtained by Fourier space analysis. That confirms the possibilities of the photothermal method for the longitudinal thermal diffusivity measurement of mural paintings.

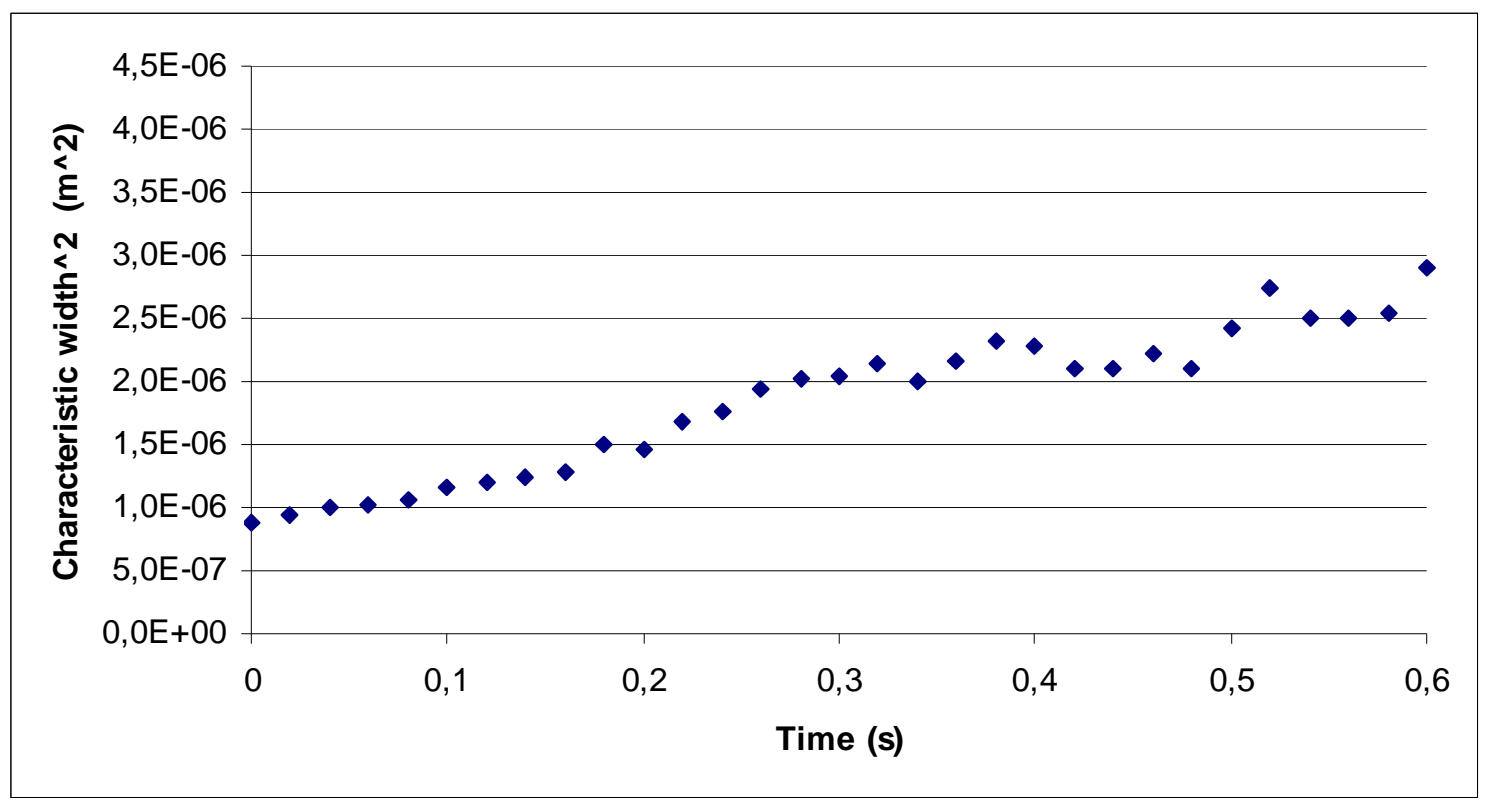

Figure 6: Temporal evolution of the characteristic radius of the photothermal signature of the laser spot 


\section{Conclusion}

In this work, we studied the possibilities of the stimulated infra-red thermography for an in situ longitudinal thermal diffusivity measurement of mural painting.

We presented first the principle of the measurement method retained for the study. It is based on the temporal analysis of the characteristic radius of the photothermal signature of a laser excitation.

We present then the experimental device developed for the study.

We showed finally, experimentally, that the method allows a good estimation of the longitudinal thermal diffusivity of a block of plaster and of a partial copy of the "Saint Christophe" of the "Campana" collection of the "Louvre".

These experimental results are encouraging because seems to permit the photothermal characterization of mural paintings.

They ask now to be generalized and being implemented for analysis of real works of art. Studies going in this direction are in progress.

\section{References}

[1] G. Szatanik, Etude et restauration d'une peinture murale représentant Saint Christophe (collection Campana, Louvre). Essai d'application de la thermographie infrarouge stimulée pour l'examen du support. Institut national du patrimoine, département des restaurateurs, 2004

[2] J.C. Candoré : Détection et caractérisation de défauts par thermographie infrarouge stimulée : Application au contrôle d'œuvres d'art. Phd thesis, Reims, jui 2010.

[3] G. Szatanik, M. Gerard, J.L.Bodnar : La thermographie infrarouge stimulée pour l'examen des enduits dans les peintures murales : Application au Saint Christophe portant l'enfant Jésus. Journal de Conservation et restauration (coré), $\mathrm{n}^{\circ} 17$, décembre 2006, pp $64-70$.

[4] J.C. Candoré, J.L Bodnar, V. Detalle and P. Grossel, Assistance to the restoration of historical frescoes by stimulated infrared thermography, QIRT 2010, pp 660-670.

[5] J. C. Candoré, J.L Bodnar, V. Detalle, P. Grossel : Non destructive testing of work of art by stimulated infrared thermography. European physical journal Applied Physics, 2012, vol 57, 02, pp $21002-210011$.

[6] J.C. Candoré, J.L. Bodnar, V. Detalle, B. Remy et P. Grossel: Approach of the measurement of the thermal of thermal diffusivity of mural painting by front face photothermal radiometry, Journal of physics, 214, (2010), 012094.

[7] J.C. Candoré, J.L Bodnar, V. Detalle, P. Grossel : Characterization of defects situated in a fresco by stimulated infrared thermography, European physical Journal Applied Physics, 2012, vol 57, 01, pp 11002 -11008.

[8] W Parker, Flash method of determining thermal diffusivity, JAP, 32, (9), 1961, 1679-1684

[9] A. Degiovanni, G. Sinicki and M. Laurent : Heat pulse thermal diffusivity measurements, thermal conducvity 18, New Ork, plenum press, 1984, pp 537-551

[10] F. Cernuschi, A. Russo, L. Lorenzoni and A. Figari, in plane thermal diffusivity evaluation by infrared thermography, review of scientific instruments, vol 72, ${ }^{\circ} 10$, oct 2001 